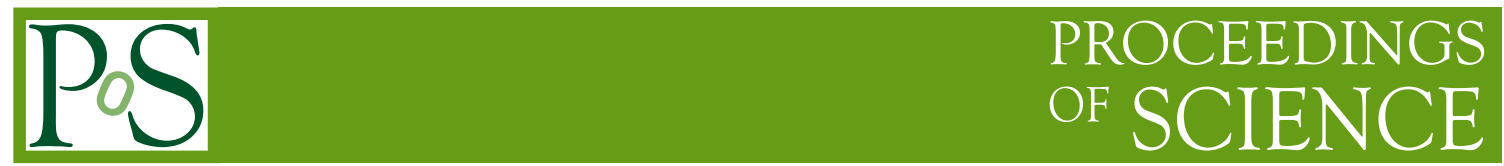

\title{
Higgs fiducial and differential measurements at ATLAS and CMS
}

\author{
Roberto Seidita ${ }^{a, b, *}$ on behalf of the ATLAS and CMS collaborations \\ ${ }^{a}$ Dipartimento di Fisica e Astronomia, Università degli Studi di Firenze, \\ Via Sansone 1, Sesto Fiorentino (FI), Italy \\ ${ }^{b}$ INFN Sezione di Firenze, \\ Via Sansone 1, Sesto Fiorentino (FI), Italy \\ E-mail: roberto.seidita@cern.ch
}

Recent differential cross section measurements in the Higgs sector published by the ATLAS and CMS collaborations are presented, exploiting the full data set from Run 2 of the LHC. Results cover an extensive set of observables, production modes and final states. In particular, the measurements that are presented include Higgs boson decays to a pair of $\mathrm{W}$ or $\mathrm{Z}$ bosons, a pair of photons, a pair of $\tau$ leptons and a pair of $b$ quarks. All results are found to be in good agreement with the Standard Model of particle physics.

The Ninth Annual Conference on Large Hadron Collider Physics - LHCP2021

7-12 June 2021

Online

${ }^{*}$ Speaker 


\section{Introduction}

Since the discovery of a new scalar resonance, compatible with the Higgs boson predicted by the Standard Model (SM) of particle physics [1-3], by the ATLAS [4] and CMS [5] collaborations, its properties have been measured with ever increasing precision. One particularly important class of measurements is that of differential and fiducial production cross sections: these allow to extract information on the coupling structure of the Higgs sector of the SM in a way that relies on relatively few a priori assumptions; moreover all measurements discussed in this report are unfolded to particle level by correcting the observable distributions for distortions caused by the finite resolution of the detectors. These two factors combined make such results directly comparable with predictions from theory, and thus serve both as a benchmark for the SM itself as well as a test for possible Beyond the SM (BSM) theories. This overview covers differential and fiducial Higgs boson production cross section measurements in the $\mathrm{H} \rightarrow \mathrm{ZZ}[6,7], \mathrm{H} \rightarrow \gamma \gamma$ [8, 9], $\mathrm{H} \rightarrow \mathrm{WW}$ [10], $\mathrm{H} \rightarrow \mathrm{b} \overline{\mathrm{b}}$ [11, 12] and $\mathrm{H} \rightarrow \tau \tau$ [13] decay channels. All results discussed exploit the entire data set gathered by the detectors during Run 2 of the LHC, corresponding to an integrated luminosity of $139 \mathrm{fb}^{-1}$ and 137 $\mathrm{fb}^{-1}$ for the ATLAS and CMS experiments respectively, with the exception of the CMS H $\rightarrow \gamma \gamma$ result which makes use of data gathered in 2016 for an integrated luminosity of $36 \mathrm{fb}^{-1}$.

\section{Observables}

An extensive set of observables is covered, providing information on different physics aspects: the transverse momentum $\left(p_{T}^{H}\right)$ and pseudorapidity $\left(\eta_{H}\right)$ of the Higgs boson, as well as the number of hadronic jets produced in association $\left(N_{j e t s}\right)$ and the invariant mass $\left(m_{j j}\right)$, pseudorapidity $\left(\Delta \eta_{j j}\right)$ and azimuthal angle $\left(\Delta \phi_{j j}\right)$ separation of the leading dijet pair probe perturbative QCD calculations, radiative corrections, coupling of the Higgs boson to light quarks and the CP structure of the Higgs sector; observables related to associated hadronic jets, such as their transverse momenta $\left(p_{T}^{j}\right)$, probe radiative QCD emission patterns and resummation effects; finally, decay related observables such as the invariant mass of the dilepton pairs in $\mathrm{H} \rightarrow \mathrm{ZZ} \rightarrow 4 \ell$ decays probe electroweak corrections.

The extent of the data set gathered by the ATLAS and CMS detectors during Run 2 of the LHC has also opened the possibility to perform multi-differential cross section measurements. While still statistically constrained as of now, such measurements will become increasingly relevant once data from Run 3 and High Luminosity LHC will be available.

\section{Bosonic channels}

Analyses targeting events in which the Higgs boson decays to either a pair of $W$ or $Z$ bosons or a pair of photons benefit from relatively clean and well reconstructed final states. This is reflected in a good sensitivity across the accessible phase space. Results from both ATLAS and CMS in the $Z Z$ final state include cross sections as a function of $p_{T}^{H},\left|y_{H}\right|, N_{\text {jets }}$ and the transverse momentum of the leading jet produced in association with the Higgs boson $p_{T}^{j_{1}}$. The ATLAS results expand on this set with a number of other observables, including the invariant masses of the two dilepton pairs in the final state, the invariant mass $\left(m_{j j}\right)$ pseudorapidity $\left(\Delta \eta_{j j}\right)$ and azimuthal angle separation $\left(\Delta \phi_{j j}\right)$ of the leading dijet system and a number of angular variables characterizing the decay of 
the Higgs boson. Uncertainties on the differential cross sections range from $10 \%$ to around $75 \%$ in the least sensitive bins, with the statistical component being the dominant contribution. Results also include a set of double-differential cross sections measured by the ATLAS collaboration as a function of two-dimentional observables such as $\left(p_{T}^{H},\left|y_{H}\right|\right)$ and $\left(p_{T}^{H}, N_{\text {jets }}\right)$. Results from the ATLAS collaboration are compared to predictions from MadGraph5_aMC@NLO [14] with the FXFX merging scheme, NNLOPS [15], RADISH [16] and NNLO]ET [17]; results from CMS are compared to predictions from POWHEG 2.0 [18] and NNLOPS. The agreement with the SM is found to be good in all distributions.

Similarly good overall sensitivity is achieved in the diphoton final state. Both collaborations present measurements of $p_{T}^{H},\left|y_{H}\right|, N_{j e t s}, p_{T}^{j_{1}}, m_{j j}$ and $\Delta \phi_{j j}$, with the CMS results also including transverse momenta and rapidity of the two leading jets, the pseudorapidity gap between the leading jets as well as the rapidity and azimuthal angle separation between the diphoton system and the leading jet. Results from the ATLAS collaboration are compared to POWHEG NNLOPS normalized to $\mathrm{N}^{3} \mathrm{LO}(\mathrm{QCD})+\mathrm{NLO}(\mathrm{EW})$, while results from the CMS collaboration are compared to MadGraph5_aMC@NL0 reweighted to match the NNLOPS prediction. In all cases the agreement with the SM is found to be good, with uncertainties ranging from $\sim 10 \%$ in the most sensitive bins to $\sim 50 \%$ in the least sensitive ones in the ATLAS results, and between $\sim 35 \%$ and $\sim 200 \%$ in the CSM results. In all cases the dominant contribution to the overall uncertainty is of statistical nature, especially in the CMS results which rely on data gathered in 2016 for and integrated luminosity of $36 \mathrm{fb}^{-1}$.

Finally, the CMS collaboration has performed a differential cross section measurement in $p_{T}^{H}$ and $N_{\text {jets }}$ targeting events in which the Higgs boson decays to a pair of $W$ bosons. Compared to the other bosonic channels described above, the $W W$ channel benefits from a larger branching fraction, but suffers from the presence of neutrinos in the final state (due to the dacay of the $W$ bosons) reducing experimental sensitivity especially at lower values of $p_{T}^{H}$. This combination makes this channel the only bosoinc one in which systematic uncertainties play a role comparable to the statistical contribution. For the same reason, given the larger detector resolution effects at low $p_{T}^{H}$ in this channel, a regularisation constraint is applied within the unfolding procedure in the $p_{T}^{H}$ measurement. Overall uncertainties on the differential cross sections range from $\sim 15 \%$ to $\sim 100 \%$. Results are compared to MadGraph5_aMC@NLO and found to be in good agreement with the SM.

\section{Fermionic channels}

In the space of differential measurements of the Higgs boson's production cross section fermionic channels play a complementary role to bosonic ones, in that where the latter provide good sensitivity on a wide range of different observables, the former contribute significantly in specific regions of phase space. Such is the case for the $b \bar{b}$ final state especially: while at low $p_{T}^{H}$ this channel suffers from an overwhelming QCD induced background, its 58\% branching fraction makes it crucial in the very high (i.e. above $\sim 500 \mathrm{GeV}$ ) $p_{T}^{H}$ regime, where the overall production cross section naturally decreases. At these energies the two b-quark induced jets (b-jets) coalesce into a single, large radius jet with a substructure compatible with two sub-jets. This is commonly referred to as the boosted topology, and its peculiar kinematic features can be exploited to separate the $\mathrm{H} \rightarrow \mathrm{b} \overline{\mathrm{b}}$ signal from the background. The two experiments report measurements of the $p_{T}^{H}$ 

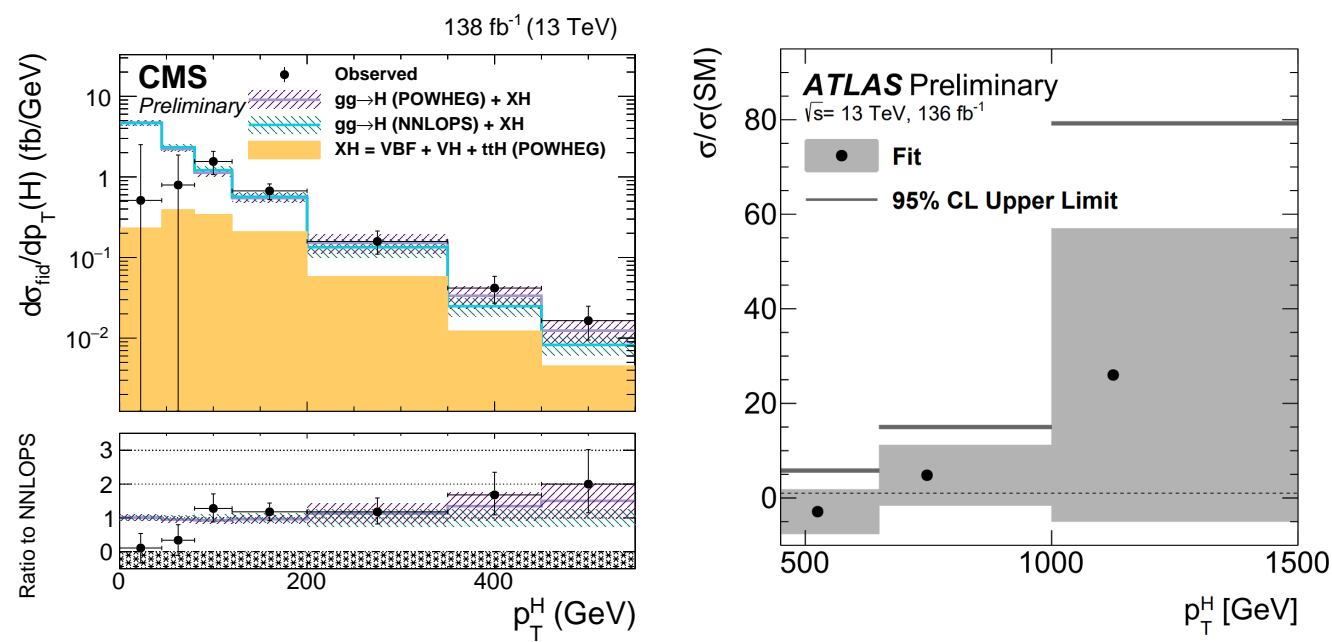

Figure 1: The $p_{T}^{H}$ spectrum as measured by CMS in the $\mathrm{H} \rightarrow \tau \tau$ channel [13] (left), and by ATLAS in the $\mathrm{H} \rightarrow \mathrm{b} \overline{\mathrm{b}}$ channel [11] (right).

spectrum, starting from $300 \mathrm{GeV}$ and $450 \mathrm{GeV}$ for the CMS and ATLAS results respectively, with the unique feature of having sensitivity for transverse momenta of the order of $1 \mathrm{TeV}$. Uncertainties are statistically dominated, and results are found to be compatible with predictions from the SM.

Finally, the CMS collaboration reported on the first Higgs boson differential cross section measurement in the $\tau \tau$ final state. Similarly to the $b \bar{b}$ channel, this analysis benefits from a large branching fraction of $6 \%$, making it competitive with bosonic channels especially at high $p_{T}^{H}$ and larger jet multiplicities. All decay combinations of the pair of $\tau$ leptons are taken into consideration, excluding purely leptonic ones were the final state leptons are of the same flavor. Results include $p_{T}^{H}$, $N_{\text {jets }}$ and $p_{T}^{j_{1}}$ spectra. Similarly to the previously discussed $\mathrm{H} \rightarrow \mathrm{WW}$ analysis, a regularisation term is added to the unfolding procedure to prevent large fluctuations due to detector effects. Uncertainties range from order $20 \%$ to order $100 \%$.

\section{Conclusions}

The extensive data set gathered by the ATLAS and CMS detectors during Run 2 of the LHC has enabled a wide range of detailed measurements of the properties of the Higgs boson, two examples of which are shown in Fig. 1. The available fiducial and differential results cover a wide range of observables in different decay channels of the Higgs boson, serving both as precision benchmarks for the SM as well as probes for possible deviations from it, with many BSM theories predicting discrepancies in the tails of differential cross sections. The combination of bosonic and fermionic decays allows to cover a large portion of phase space, with reach up to the TeV scale. The uncertainties in most analyses are currently constrained by the statistical power of the Run 2 data set, and will greatly benefit from Run 3 and the High Luminosity phase of the LHC. The increased amount of available data will enhance the reach of all channels and enable finer measurements (e.g. narrower bins in the observables, more and more precise multi-dimentional diferential cross sections), further boosting the sensitivity to new physics. Finally, all results discussed are unfolded to particle level, enabling direct comparison with theory. 


\section{References}

[1] ATLAS collaboration, Observation of a new particle in the search for the Standard Model Higgs boson with the ATLAS detector at the LHC, Physics Letters B 716 (2012) 1.

[2] CMS collaboration, Observation of a new boson at a mass of $125 \mathrm{GeV}$ with the CMS experiment at the LHC, Physics Letters B 716 (2012) 30.

[3] CMS collaboration, Observation of a new boson with mass near $125 \mathrm{GeV}$ in pp collisions at $\sqrt{s}=7$ and 8 TeV, Journal of High Energy Physics 2013 (2013) 81.

[4] ATLAS collaboration, The ATLAS Experiment at the CERN Large Hadron Collider, JINST 3 (2008) S08003.

[5] CMS collaboration, The CMS Experiment at the CERN LHC, JINST 3 (2008) S08004.

[6] ATLAS collaboration, Measurements of the Higgs boson inclusive and differential fiducial cross sections in the $4 \ell$ decay channel at $\sqrt{s}=13 \mathrm{TeV}$, The European Physical Journal C 80 (2020) 942.

[7] CMS collaboration, Measurements of production cross sections of the Higgs boson in the four-lepton final state in proton-proton collisions at $\sqrt{s}=13 \mathrm{TeV}$, Eur. Phys. J. C 81 (2021) 488 [2103.04956].

[8] CMS collaboration, Measurement of inclusive and differential Higgs boson production cross sections in the diphoton decay channel in proton-proton collisions at $\sqrt{s}=13 \mathrm{TeV}$, Journal of High Energy Physics 2019 (2019) 183.

[9] ATLAS collaboration, Measurements and interpretations of Higgs-boson fiducial cross sections in the diphoton decay channel using $139 \mathrm{fb}^{-1}$ of pp collision data at $\sqrt{s}=13 \mathrm{TeV}$ with the ATLAS detector, Tech. Rep. ATLAS-CONF-2019-029, CERN, Geneva (Jul, 2019).

[10] CMS collaboration, Measurement of the inclusive and differential Higgs boson production cross sections in the leptonic WW decay mode at $\sqrt{s}=13 \mathrm{TeV}$, Journal of High Energy Physics 2021 (2021) 3.

[11] ATLAS collaboration, Study of Higgs-boson production with large transverse momentum using the $H \rightarrow b \bar{b}$ decay with the ATLAS detector, Tech. Rep. ATLAS-CONF-2021-010, CERN, Geneva (Mar, 2021).

[12] CMS collaboration, Inclusive search for highly boosted Higgs bosons decaying to bottom quark-antiquark pairs in proton-proton collisions at $\sqrt{s}=13 \mathrm{TeV}$, Journal of High Energy Physics 2020 (2020) 85.

[13] CMS collaboration, Measurement of the inclusive and differential Higgs boson production cross sections in the decay mode to a pair of $\tau$ leptons, Tech. Rep. CMS-PAS-HIG-20-015, CERN, Geneva (2021). 
[14] M. Wiesemann, R. Frederix, S. Frixione, V. Hirschi, F. Maltoni and P. Torrielli, Higgs production in association with bottom quarks, Journal of High Energy Physics 2015 (2015) 132.

[15] K. Hamilton, P. Nason, E. Re and G. Zanderighi, NNLOPS simulation of Higgs boson production, Journal of High Energy Physics 2013 (2013) 222.

[16] A. Banfi, P.F. Monni, G.P. Salam and G. Zanderighi, Higgs- and Z-boson Production with a Jet Veto, Phys. Rev. Lett. 109 (2012) 202001.

[17] X. Chen, T. Gehrmann, E. Glover and M. Jaquier, Precise QCD predictions for the production of Higgs + jet final states, Physics Letters B 740 (2015) 147.

[18] P. Nason, A New Method for Combining NLO QCD with Shower Monte Carlo Algorithms, Journal of High Energy Physics 2004 (2004) 040. 